
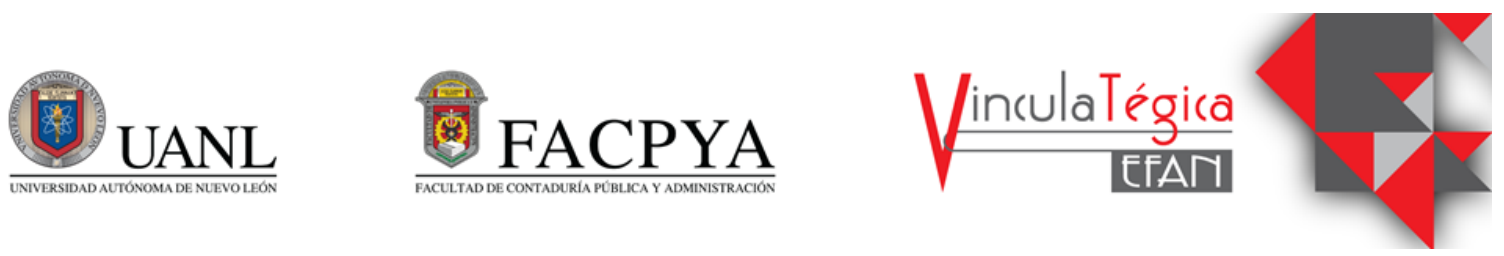

\title{
Factores sociales en la responsabilidad social de las empresas del sector turismo
}

\author{
Óscar Ernesto Hernández Ponce ${ }^{1}$, Javier Saucedo Monarque ${ }^{2}$, Edith Patricia Borboa \\ Álvarez ${ }^{3}$ \\ ${ }^{1}$ Instituto Tecnológico de Sonora, oscar.hernandez@itson.edu.mx, ITSON Unidad Guaymas, 6222210032 \\ ${ }^{2}$ Instituto Tecnológico de Sonora, jsaucedo@itson.edu.mx, ITSON Unidad Guaymas, 6222210032 \\ ${ }^{3}$ Instituto Tecnológico de Sonora, epborboa@itson.edu.mx, ITSON Unidad Guaymas, 6222210032
}

\author{
Información del artículo revisado por pares \\ Fecha de aceptación: junio-2021 \\ Fecha de publicación en línea: diciembre-2021 \\ DOI: https://doi.org/10.29105/vtga7.1-134
}

\section{Resumen}

El presente estudio tuvo como objetivo identificar los factores sociales que insiden significativamente en la Responsabilidad Social de las empresas turísticas de Guaymas, Sonora y Bahías de Huatulco, Oaxaca. El método utilizado fue a través de un estudio descriptivo con un corte cualitativo, donde se evaluó los diferentes factores sociales de la responsabilidad social; y con base en ello, se buscó determinar cuáles son los factores de la dimensión social de la responsiva social empresarial que inciden en las empresas del sector turístico. Con base en los resultados, se categorizaron cuatro factores sociales que inciden positivamente en las empresas del sector turístico en los destinos antes mencionados, los cuales son: 1) Condiciones laborales e inclusión, 2) Flexibilidad laboral y programas de formación, 3) Proyectos sociales e igualdad, 4) Participación social y voluntariado.

Palabras clave: Factores sociales, Responsabilidad Social, Turismo

\section{INTRODUCCIÓN}

En los últimos años las empresas han buscado la forma de generar un impacto positivo en la sociedad y medio ambiente; este cambio se ha convertido en una obligación de adoptar estrategias que produzcan una armonía social, empresarial, gubernamental o familiar y que a

\begin{abstract}
The objective of this reseach was to identify the social factors that significantly influence the Social Responsibility of the tourist companies of Guaymas, Sonora and Bahías de Huatulco, Oaxaca. The method used was through a descriptive study with a qualitative cut, where the different social factors of social responsibility were evaluated; Based on this, it was sought to determine which are the factors of the social dimension of the business social responsive that affect companies in the tourism sector. Based on the results, four social factors that positively affect companies in the tourism sector in the aforementioned destinations were categorized, which are: 1) Labor conditions and inclusion, 2) Labor flexibility and training programs, 3) Social projects and equality, 4) Social participation and volunteering.
\end{abstract}

Keywords: Social factors, Social Responsibility, Tourism

JEL: M1, M14, L83.

su vez contribuyen en la disminución de los impactos negativos provocados hacia la sociedad, los cuales según Viteri (2010) son: problemas ambientales, pobreza, desigualdad, atención de salud, hambruna, desnutrición, y analfabetismo. 
Han surgido diversos estudios enfocados en los problemas generados en el entorno por el desarrollo de activiadades económicas, los cuales se pueden encuadrar en el termino de la Responsabilidad Social; y dichos estudios, han buscado dar respuesta a través de soluciones y normativas en las áreas de interacción social (Vélez \& Cano, 2016).

El surgimiento de la Responsabilidad Social (RS) en Europa y Estados Unidos, por presiones por parte de la sociedad ante la producción de productos con alcohol y la venta de tabaco, los cuales generaban daños en la salud social; a partir de esta situación, las empresas buscaron una forma adecuada de administración de su riqueza y al mismo tiempo tratar de aminorar las necesidades de los individuos más pobres (Romo, 2016).

Los individuos han decidido comprometerse a identificar los diversos problemas de interés público, para generar estrategias que solucionen las problemáticas y que a su vez mejoren la situación actual de la sociedad, con base a factores como la sustentabilidad, ética, transparencia y cooperación, siendo la meta común alcanzar el desarrollo sustentable del ser humano y del entorno (Viteri, 2010).

A partir de esas situaciones, han surgido diferentes proyectos internacionales que han representado importantes avances para el fortalecimiento de la Responsabilidad Social desde el enfoque económico, social y medioambiental. Los documentos internacionales de referencia son: el Pacto Mundial de las Naciones Unidas para la Responsabilidad Social Corporativa; las Líneas Directrices para Empresas Multinacionales de la OCDE y el Libro Verde aprobado por la Comisión Europea en 2001 (Camacho, 2015).

La Responsabilidad Social consiste, en lograr objetivos económicos, la aportación de beneficios a la sociedad; con principios éticos, cívicos y de la normatividad legal; así como la formación de los recursos humanos, en temas de desempeño laboral, mejoramiento personal, familiar y social; asegurando el ambiente laboral y el bienestar de las personas, así como la consideración de las decisiones en función de cuestiones éticas y ambientales (Camacho, 2015).

La Comisión Europea (2001), "las empresas que prestan una atención adecuada a los aspectos sociales y medioambientales indican que tales actividades pueden redundar en una mejora de sus resultados y generar crecimiento y mayores beneficios". Además, una empresa comprometida con la sociedad deriva múltiples resultados, tanto directos, como el incremento del compromiso de los trabajadores y la productividad, e indirectos como la mejora de la imagen corporativa y mayores ventajas competitivas en el mercado.

Cabe destacar que la Responsabilidad Social tiene un enfoque tanto interno y como externo. En lo interno, está el aspecto de los derechos de los trabajadores, la aplicación de la legislación laboral y de las normas de la Organización Internacional del Trabajo, así como, la forma en que las empresas deben enfocar los esfuerzo en el desarrollo personal y profesional de los recursos humanos, con la mejora de las condiciones y calidad de vida, las funciones laborales, permitiendo un equilibrio entre la vida familiar y laboral; y con ello, fortalecer sus relaciones y establecer un compromiso permanente con el respeto a los derechos humanos y laborales (Jaramillo, 2011).

Desde el punto de vista externo, la Responsabilidad Social se extiende hasta los habitantes de la localidad, trabajadores, accionistas, socios comerciales y proveedores, consumidores, autoridades públicas y Organizaciones No Gubernamentales (ONG), las cuales defienden y protegen los intereses de las comunidades locales y el medio ambiente (Comisión Europea, 2001).

Por lo antes mencionado, la dimensión social, significa que las empresas 
sean responsables por los efectos sociales, tanto negativos como positivos, que sus acciones puedan provocar. Szczuka (2015), menciona que las empresas como actores sociales, deben prestar especial atención al servicio de las comunidades tanto internas como externas, responder a sus necesidades, expectativas, derechos y demandas para mejorar su bienestar. $\mathrm{Y}$ es precisamente, esta dimensión un factor clave para que las empresas establezcan relaciones con la sociedad; su principal objetivo es que las organizaciones trabajen para mejorar las condiciones de la sociedad e integrar las preocupaciones sociales en sus operaciones comerciales, considerando el alcance que tienen sus acciones en la comunidad (Nasrullah \& Rahim, 2014).

Específicamente en el sector turismo, la Responsabilidad social es de vital importancia, debido a que los servicios turísticos dependen significativamente de la buena reputación que tienen las empresas; por lo que es de esperarse que estas cumplan con ciertas condiciones que garanticen un correcto control y una adecuada respuesta hacia sus acciones, que les permita aumentar su credibilidad ante la opinión pública, ofreciendo confianza y seguridad a los turistas (Ledesma, 2009).

Las empresas que practican la Responsabilidad Social fortalecen y aportan al desarrollo de una actividad sostenibles y al considerar las necesidades de las poblaciones y el medioambiente (Salnivoka et al, 2021). Las actividades turísticas se han redirigido hacia la búsqueda de acciones que permitan mejorar los beneficios y dismunuir los problemas provocados por el fenómeno turístico.

Asimismo, el desarrollo sustentable y la Responsabilidad Social proveen las bases para una nueva lógica en el aspecto económico y el desarrollo social, donde las acciones deben estar orientadas a mejorar la distribución igualitaria de los ingresos, la protección de la sociedad indígena y del patrimonio cultural, y promueve el involucramiento de los habitantes en los planes de desarrollo (Jucan \& Jucan, 2010).

En México el tema de la Responsabilidad Social es nuevo, y hasta cierto punto limitado y confuso por parte de los altos mandos ejecutivos y por los consumidores en general; sin embargo, los empresarios no solo consideran la Responsabilidad Social como donaciones filantrópicas, sino también como una acción de mercadep, pero no han adquirido el concepto lo suficientemente fuerte como para cambiar los paradigmas administrativos de las empresas. (Romo, 2016).

Fue en los años sesenta cuando en México se creó la Unión Social de Empresarios Mexicanos (USEM) por parte de la inciativa de la Unión de Empresarios Católicos fundada; y en 1963 surge la Fundación Mexicana para el Desarrollo Social (FMDR), por parte de empresarios católicos con el proposito de dar solución a problemas de zonas marginales por medio de estrategias de desarrollo social con actividades activas por parte de los involucrados. Además, se funda el Consejo Coordinador Empresarial (CEE), donde se agruparon a las cámaras de comercio, de la industria, de transformación, entre otras, con el fin proporcionar acciones y normas para la adpción de la Responsabilidad Social por parte de las empresas (Camacho, 2015).

En el año 2015, la SECTUR actualizó el Directorio nacional de empresas con distintivo "S" vigentes, donde muestra las 103 empresas turísticas que han sido reconocidas en este ámbito, de las cuales 7 se localizan en el Estado de Oaxaca; 8 en Guerrero; 32 en Quintana Roo; 6 en Nayarit; 3 en Jalisco; 2 en Sonora; 7 en Baja California Sur; 2 en Colima; 9 en la Ciudad de México; 4 en Puebla; 2 en Veracruz; 1 en Morelos; 3 en Yucatán; 5 en Sinaloa; 1 en Coahuila; 1 en San Luis Potosí; 1 en Guanajuato; 1 en Zacatecas; 1 en Chihuahua; 3 en Chiapas; 2 en el Estado de México; 1 en Querétaro y 1 en Nuevo 
Vallarta. (Secretaria de Turismo, 2017).

Los datos anteriores, figuran un comportamiento positivo y de iniciativa por parte de las empresas mexicanas del sector turístico, donde el turismo juega un papel importante en el fortalecimiento del respeto a las culturas de cada destino turístico, buscando la igualdad entre hombres y mujeres, la conservación del patrimonio cultural, la Responsabilidad Social corporativa y la protección de los niños (OMT, 2012).

En este contexto, los indicadores juegan un papel importante al ser los principales instrumentos cuantitativos utilizados durante el proceso de determinación de parámetros relativos a las actividades desempeñadas por las empresas, su Responsabilidad Social y Sustentabilidad. Además, estos son parte fundamental en cualquier proceso de gestión y planificación organizacional, ya que permiten generar información, son consistentes metodológicamente, válidos, y de fácil aplicación y comunicación (López, Torres, Font \& Serrano, 2018).

Finalmente, es importante analizar cómo se está adoptando la Responsabilidad Social en el sector turístico, así como las acciones que ejecutan las diferentes empresas del sector turístico de la región de Guaymas y Bahías de Huatulco, para la generación de indicadores que condicionen y determinen la dimensión social de Responsabilidad Social en las empresas turísticas para que estas puedan comprometerse en el cumplimiento de estos y que, a su vez, les permiten ser reconocidas en su labor, iniciativa y compromiso por contribuir de manera positiva a su entorno, y obtener un impacto competitivo positivo en el mercado.

Los impactos socioculturales del turismo han sido descritos como las formas en que el turismo y los viajes transforman los sistemas de valores sociales y colectivos, patrones de comportamiento, estructuras comunitarias, estilo y calidad de vida. Además, son difíciles de categorizar debido a que las dimensiones sociales, culturales, ambientales y económicas de las comunidades locales no son facil de distinguir (Mendoza, et al, 2011) (Gallardo, 2019).

Los factores como la catergoria de turistas, el nivel de desarrollo turístico y las condiciones económicas, socioculturales y políticas de la comunidad local; determinan el grado del impacto social con base en el cambio generado por la actividad turística en la comunidad y de la capacidad resilencia que se tenga para recibir dichos cambios (Mendoza et al., 2011).

La Responsabilidad Social es un tema muy extenso, del cual es importante analizar cada una de sus dimensiones con sumo detenimiento para comprender cómo funcionan, sus respectivos indicadores, así como los beneficios competitivos que provee a las empresas; específicamente del sector turístico, el desarrollar acciones que les permitan generar un impacto socialmente responsable en el entorno. Por este motivo se plantea como pregunta de investigación lo siguiente:

\section{¿Cuáles son los factores sociales que insiden significativamente en la Responsabilidad Social de las empresas turísticas?}

Para fines de esta investigación y buscando dar respuesta al planteamiento del problema se estableció como objetivo el siguiente:

Identificar los factores sociales que
insiden significativamente en la
Responsabilidad Social de las empresas
turísticas de Guaymas/ San Carlos, Sonora
y Bahías de Huatulco, Oaxaca.

\section{MARCO TEÓRICO}

La ISO 26000 (2011), señala 4 principales 
características de la responsabilidad social:

La primera característica es la voluntad que poseen las organizaciones de incorporar, en su toma de decisiones, temas del ámbito social y ambiental, asimismo como rendir cuentas por los impactos provocados a la sociedad y el medio ambiente; lo que implica una actuación ético que aporte al desarrollo sostenible, cumpliendo con la legislación aplicable y sea coherente con la normativa internacional de comportamiento.

La segunda característica es la expectativa de la sociedad, las empresas e instituciones también deben comprender las más amplias expectativas de la sociedad, así como el respeto por el cumplimiento de la normatividad vigente y de las obligaciones de cada individuo, ya que estas obligaciones representan y promueven los valores éticos.

La tercera característica tiene que ver con las acciones de los involucrados, lo cual es fundamental para la responsabilidad social. Una empresa debería determinar acciones que aumente el intereses en la toma de decisiones con el fin de que todos puedan comprender sus impactos y cómo abordar las problemáticas sociales.

La cuarta característica es la integración de la responsabilidad social con la rendición de cuentas en todos los niveles de la organización. Debido a que los impactos de las decisiones o actividades de una organización pueden influir en las relaciones con otras organizaciones, estas deben aprender a trabajar con otras, para poder abordar sus responsabilidades; se pueden incluir organizaciones pares, competidores (mientras tenga cuidado de evitar conductas anticompetencia), otras partes de la cadena de valor o cualquier otro grupo relevante dentro de la esfera de influencia de la organización.

Camacho (2015), menciona que es importante que las empresas fundamenten su Responsabilidad Social en principios que sirvan como un marco de actuación ético, responsable y adecuado con respecto a las exigencias de la sociedad. Por ello, los principios básicos de Responsabilidad Social son: respeto a la dignidad de las personas, justicia, solidaridad, subsidaridad, trabajo, legitimidad institucional, responsabilidad pública y discreción empresarial.

Se identifican tres niveles esenciales dentro de la Responsabilidad Social, los cuales son: nivel primario: Este se constituye como la primera etapa para el desarrollo de estrategias sustentables; nivel secundario: es donde la empresa establece los vínculos entre los empleados y la comunidad; y finalmente el nivel terciario: en este nivel la empresa adquiere responsabilidades que no son obligatorias, como la realización de donaciones a poblaciones vulnerables, contribuciones a la educación, creación de entornos deportivos para contribuir a la salud y bienestar de la comunidad y el desarrollo de actividades voluntarias donde se eleva la productividad de la empresa y se genere un beneficio para la comunidad. (Vélez \& Cano, 2016).

La actividad turística, de acuerdo con Mercado y Palmerín (2012), se ha convertido en una oportunidad para el crecimiento y desarrollo para los destinos turísticos, lo que les permite aumentar la calidad y el nivel de vida de sus residentes, con la generación de empleos, ingresos para la sociedad, generación de empresas, con actividades de participación social.

Con respecto al tema de inclusión laboral, Martínez (2013), menciona que los negocios inclusivos muestran que las oportunidades de crecimiento e innovación se presentan tanto para el lado de la demanda como para el de la oferta. Les permite a las empresas tener acceso a bienes básicos, a servicios, a empleo e ingresos, entre otros beneficios.

Asimismo, menciona que en el caso de las empresas turísticas que son inclusivas, estas ayudan a personas de escasos recursos a mejorar su economía famliar, fomentan la buena relación y productividad de los 
trabajadores, y se fortalece la relación con clientes, haciéndolos leales. Además, las empresas aportan importantes beneficios; ya que se fortalece la eficiencia operativa y la nuevas fuentes de innovación y oportunidades de internacionalizar sus productos y servicios.

Por otra parte, es debido mencionar el concepto de calidad de vida laboral, el cual de acuerdo con Molina, Pérez, Lizárraga y Larrañaga (2018), es el proceso que permite a las empresas también atener a las necesidades de sus empleados, con el proposito de mejorar su vida laboral, así como su desarrollo profesional y personal, aplicando políticas justas de remuneración, compensación e incentivos, además, permitiendo contar con un ambiente de trabajo adecuado y de pertenencia, tomándolo como medio para elevar la eficiencia empresarial.

De igual manera Rueda (2011), separa la calidad de vida en cuatro bloques, el primero con un enfoque para el bienestar general del residentes de la comunidad, como lo es trabajo, educación, sanidad, vivienda y equipamientos.

En el segundo bloque se refiere a la contribución que tiene el medio en la vida de las personas, como lo es la calidad ambiental, tal como la reducción del ruido, aumento de la distribución de la calidad del agua, entre otros. El tercer bloque incluye la naturaleza psicosocial y está vinculado al ámbito interactivo del sujeto, sus relaciones familiares, relaciones interpersonales, ocio, tiempo libre y demás. El último bloque hace mención de las cuestiones de orden sociopolítico, como es la participación social, la seguridad personal y jurídica, etcétera.

Así pues, un aspecto importante es la obligación que tienen los empresarios en que empleen las medidas necesarias y dediquen sus esfuerzos a regular $y$ establecer obligaciones jurídicas, permitiendo la conservación de un medio ambiente de trabajo sano para los trabajadores (Mendizábal, 2013).

\subsection{Modelo teórico del estudio descriptivo}

A continuación se presenta el modelo teórico que permitió del desarrollo del presente estudio descriptivo.

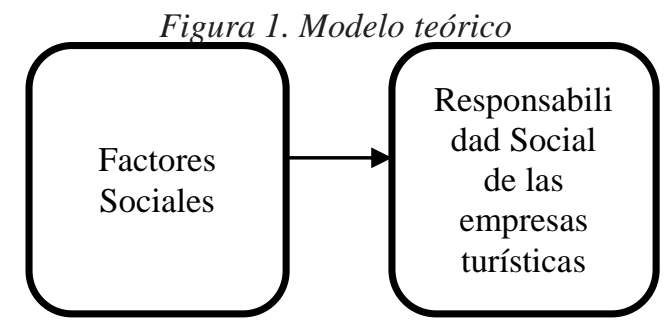

Elaboración propia

\section{MÉTODO}

El desarrollo del presente estudio fue con base en el método de estudio descriptivo con corte cualitativo y consideraron las principales empresas turísticas del Puerto de Guaymas, Sonora y Bahías de Huatulco, Oaxaca. Esto con el fin de recabar la información pertinente para la obtención de los resultados esperados. Para la región de Guaymas se consideraron aquellas empresas desde pequeñas hasta grandes empresas y cuya función es brindar los servicios de hospedaje y de alimentos y bebidas. Mientras que, en la región de Bahías de Huatulco, se tomaron como sujetos de estudio a las empresas que pertenecen al sector de servicios y de comercio, tales como empresas de hospedaje, alimentos y bebidas, tiendas de souvenirs y artesanías, tiendas de ropa y arrendadoras.

Para determinar la población de empresas de hospedaje y restaurantes de la región de Guaymas y San Carlos, se tomó como referencia el censo de 2017 realizado por INEGI, el cual muestra un total de 91 empresas de alimentos y bebidas y 33 establecimientos de hospedaje, dando un total de 124 empresas.

Asimismo, para la región de Bahías de Huatulco, se tomó como referencia el censo de 2017 realizado por INEGI, el cual 
muestra un total de 111 empresas de hospedaje, 227 empresas de alimentos y bebidas, 11 agencias de viajes, 7 empresas arrendadoras y 27 empresas de spa, dando un total de 376 empresas.

El tipo de muestreo fue probabilístico, aleatorio y estratificado, donde se hicieron grupos estandares por prestación de servicio y grio turístico y se seleccionaron con el muestreo "al azar".

Los sujetos de estudio fueron el personal de nivel gerencial de las empresas de servicios turísticos de Guaymas y Huatulco, y se obtuvo como muestra 242 encuestas, las cuales se distribuyeron de la siguiente manera (tabla 1).

Tabla 1. Muestra

Fuente: Elaboración propia

Para el diseño del instrumento de recolección de datos, primeramente, se realizó una cuantificación de las dimensiones, para posteriormente definir los ítems que conforman cada apartado de la encuesta.

Para la validación del instrumento, se llevó a cabo una prueba piloto, donde se aplicó el instrumento a una muestra de 21 sujetos y con los resultados, se determinó la fiabilidad del mismo a través del Alpha de Cronbach; el cual arrojó un resultado favorable de 0.92 ; al haber obtenido un valor mayor a 0.70 cerca de 1 , refleja un grado de confiabilidad dicho instrumento.

Para la realización de la investigación se empleó la técnica cualitativa no experimental con componente de estadística descriptiva bajo el modelo de Hernández Sampieri (2014), ya que se describe el contexto de las empresas turísticas a través del muestreo probabilístico en donde no se modifica el objeto de estudio y únicamente se describen los datos con base en datos no paramétricos.
En las técnicas se incluyeron aspectos tales como: la descripción del problema, análisis documental del marco teórico pertinente, creación de la base de datos utilizando el sistemas de SPSS Versión 21; finalmente, se organizó la información corroborando el cumplimiento de objetivos.

\begin{tabular}{cc}
\hline Lugar & $\begin{array}{l}\text { Número de } \\
\text { encuestas }\end{array}$ \\
San Carlos & 63 \\
Guaymas & 22 \\
Bahías de Huatulco & 151 \\
Otros & 6 \\
\hline Total & 242 \\
\hline
\end{tabular}

En la investigación no se construyeron situaciones, sino que se observó el fenómeno estudiado y su acción existente. Se verificó la ocurrencia de los factores sociales en la Responsabilidad Social de las empresas turísticas sin su manipulación, ni control sobre ellas.

Se llevó a cabo la aplicación del instrumento en las diferentes empresas de hospedaje y restaurantes. Primeramente, se realizó la recolección de datos en Bahías de Huatulco y posteriormente en Guaymas y San Carlos. Sin embargo, debido a la situación por COVID-19, también se recurrió al uso de formularios de Google para la recolección de información, los cuales fueron enviados por medio de correo electrónico a los diferentes sujetos de estudio.

Después del levantamiento de encuestas a los gerentes y/o propietarios de las 242 empresas prestadoras de servicios turísticos, se generó la base de datos utilizando el programa estadístico e informático conocido como "SPSS" (Statistical Product and Service Solutions) creado por la empresa IBM (versión 21). 
Se utilizó el índice de Kaiser-MeyerOlkin (KMO), previo al Analisis Factorial (ANOVA), con el fin de comparar las magnitudes de los coeficientes de correlación parcial (De la Fuente, 2011).

Para poder realizar el Analisis Factorial, se tomarón en consideración los siguientes coeficientes:

$\mathrm{KMO} \geq 0.75 \Rightarrow$ Bien;

$\mathrm{KMO} \geq 0.50 \Rightarrow$ Aceptable;

$\mathrm{KMO}<0.50 \Rightarrow$ Inaceptable.

Si el índice KMO está próximo a uno, se considera positivo e indica que se puede continuar con el análisis factorial. Pero si se obtienen valores por debajo de 0.5 , indica que la correlación entre las variables no puede ser explicada por las otras variables y en consecuencia no es factible llevar a cabo el análisis factorial (Montoya, 2007).

Con la información capturada se generaron todos bloques de resultados con base en la técnica del Análisis Factorial (AF) permitiendo la reducción de dimensiones del conjunto de variables cualitativas y observables a un nivel confirmatorio deductivo.

\section{RESULTADOS}

Los resultados encontrados referentes al lugar de levantamiento de las encuestas muestran que el lugar donde se logró un mayor número de encuestas aplicadas fue Bahías de Huatulco con un $62.4 \%$ de encuestas realizadas (tabla 2.)

De acuerdo al tipo de empresas encuestadas, se puede observar que la mayoría de las empresas son hoteles, siendo que un $30.6 \%$ de las encuestas fueron aplicadas a este tipo de empresas, ya que son un tipo de empresas que predomina en los destinos donde se aplicó el instrumento.

Por otra parte, un $28.9 \%$ de las empresas encuestadas son empresas de bienes raíces, esto debido a la variedad de empresas de arrendamiento y venta de inmuebles que existen en los destinos estudiados. También, se tiene que un $13.6 \%$ y un $12 \%$ pertenecen a empresas como restaurantes y souvenirs/

Tabla 2. Lugar de levantamiento

\begin{tabular}{lcc}
\hline Lugar & Frecuencia & $\%$ \\
\hline San Carlos & 63 & 26 \\
Guaymas & 22 & 9.1 \\
Bahías de & 151 & 62.4 \\
Huatulco & 6 & 2.5 \\
Otros & 242 & 100 \\
\hline Total &
\end{tabular}

boutiques respectivamente (tabla 3 ).

Tabla 3. Tipo de empresa

\begin{tabular}{ccl}
\hline Clasificación & Frecuencia & $\%$ \\
\hline Hoteles & 74 & 30.6 \\
Restaurantes & 33 & 13.6 \\
Souvenir y & 29 & 12 \\
boutiques & 14 & 5.8 \\
Agencias de viajes & 14 & 28.9 \\
Bienes raíces & 70 & 0.8 \\
$\begin{array}{c}\text { Dependencias no } \\
\text { gubernamentales }\end{array}$ & 2 & 7.4 \\
$\begin{array}{c}\text { Construcción y } \\
\text { ferreterías }\end{array}$ & 18 & 0.8 \\
Guías de turistas & 2 & 100 \\
\hline Total 242 &
\end{tabular}

Se obtuvó una KMO de .989 , el cual esta cercano a uno, con una significancia de .000 (tabla 4).

Tabla 4. Prueba KMO y Bartlett

\begin{tabular}{ccc}
\hline \multicolumn{2}{c}{ Medida Kaiser-Meyer-Olkin } & .989 \\
Prueba de & Chi cuadrada & 53676.160 \\
esfericidad de & gl & 1485 \\
Bartlett & Sig. & .000 \\
\hline \multicolumn{2}{c}{ Fuente: Elaboración propia }
\end{tabular}

Con los resultados, se generaron 4 modelos, los cuales los podemos traducir en los 4 factore más significativos de la 
Responsabilidad Social en las empresas turísticas, los cuales son: 1) condiciones laborales e inclusión, 2) flexibilidad laboral y progrmas de formación, 3) proyectos sociales e igualdad, 4) participación social y voluntariado (tabla 4).

\begin{tabular}{|c|c|c|c|c|}
\hline \multicolumn{5}{|c|}{ Componente } \\
\hline & 1 & 2 & 3 & 4 \\
\hline $\begin{array}{l}\text { Condiciones } \\
\text { laborales e } \\
\text { inclusión }\end{array}$ & .3463 & & & \\
\hline $\begin{array}{l}\text { Flexibilidad } \\
\text { laboral y } \\
\text { programas de } \\
\text { formación }\end{array}$ & & .2533 & & \\
\hline $\begin{array}{l}\text { Proyectos } \\
\text { sociales e } \\
\text { igualdad }\end{array}$ & & & .1160 & \\
\hline $\begin{array}{c}\text { Participación } \\
\text { social y } \\
\text { voluntariado }\end{array}$ & & & & .8820 \\
\hline
\end{tabular}

\section{CONCLUSIONES}

Se puede concluir que existen 4 factores de la Responsabilidad Social para las empresas turísticas, los cuales son: Factor 1. Condiciones laborales e inclusión, Factor 2. Flexibilidad laboral y programas de formación, Factor 3. Proyectos sociales e igualdad y el Factor 4. Participación social y voluntariado. Dichos factores, son parte fundamental para dirigir a las empresas a ser más competitivas y que a su vez demuestren su compromiso hacia con la sociedad.

Con base en los factores mencionados y que son resultado del presente estudio descritivo, se puede diseñar un índice de indicadores que pueden servir de base para las empresas turísticas del municipio de Guaymas, Sonora y Bahías de Huatulco, Oaxaca que vayan encaminadas hacia ser empresas Socialmente Responsables, en donde integren acciones responsables en sus estrategias empresariales; teniendo como finalidad principal, el tener una identidad turística de Responsabilidad Social.
A pesar de las limitaciones presentadas durante el desarrollo de esta investigación, principalmente la pandemia COVID-19, la cual no se tenía considerado en el momento de levantamiento de encuestas y que logró retrasar sin duda la investigación, se logró concluir de manera adecuada la investigación con el uso de medidas sanitarias correspondientes para la recolección de información de campo.

A partir de la justificación planteada en la investigación, se puede decir que se pudo determinar que existen indicadores de la dimensión social de Responsabilidad Social que se pueden adaptar a las empresas turísticas, por lo que es posible que más empresas del sector turístico de Guaymas/ San Carlos y Bahías de Huatulco se sumen a ser Socialmente Responsables a partir de la aplicación de indicadores sociales propuestos especialmente para empresas de este giro.

Debido a las características únicas del sector turístico debido a que se brindan, es importante destacar que se deben aplicar indicadores que estén diseñados especialmente para este tipo de empresas y que a su vez cumplan con el objetivo de Responsabilidad Social. Además, con el diseño de indicadores Sociales de Responsabilidad Social se puede contribuir hacia la mejora de la calidad de vida de los habitantes y de los trabajadores de las regiones estudiadas, a partir de que las empresas generen acciones como empresas Socialmente Responsables.

Por último, a partir del desarrollo de esta investigación se abre un campo para que se generen investigaciones similares por parte de estudiantes que deseen realizar trabajos de investigación y empresas que deseen ampliar su conocimiento con respecto al tema de Responsabilidad Social y Turismo; ya que es importante seguir actualizando la información existente de ambos conceptos.

Una recomendación a considerar es que el estudio fue descritivo-cualitativo bajo un enfoque cualitativo, donde simplemente se 
describieron los factores sociales que más empresas turísticas. insiden en la Responsabilidad Social de las 


\section{REFERENCIAS}

Camacho, J. (2015). Las normas de responsabilidad social. Su dimensión en el ámbito laboral de las empresas. Revista Latinoamericana de Derecho Social, (20), 3-29.

Comisión Europea. (2001). Libro Verde: Fomentar un marco europeo para la responsabilidad social de las empresas. [En línea]. Disponible en: https://www.europarl.europa.eu/meetdocs/committees/deve/20020122/com(2001)366_es.pdf [2020, 02 de junio]

Comisión Europea. (2006). Indicative guidelines on evaluation methods: monitoring and evaluation indicators. [En línea]. Disponible en: https://ec.europa.eu/regional_policy/sources/docoffic/2007/working/wd2indic_082006_en.pd f [2020, 05 de junio]

De la Fuente, S. (2011). Análisis factorial. Madrid: Universidad Autónoma de Madrid.

Hernández, R. (2014). Metodología de la investigación (6 $6^{\mathrm{a}}$ ed.). México: Mc Graw Hill.

Gallardo, D., Hernández, O.E., Valdez, L.E.(2019). Impact factors for the development of a competitive and sustainable tourist destination. Case: Southern Sonora Region. SCIENDO, 9(2), 3-14. https://doi.org/10.2478/ejthr-2019-0008

Jaramillo, O. (2011). La dimensión interna de la responsabilidad social en las micro, pequeñas y medianas empresas del programa EXPOPYME de la Universidad del Norte. Pensamiento y gestión, (31), 167-195.

Jucan, C., \& Jucan, M. (2010). Social responsability and susteinable development. Wseas transactions on enviroment and development, 6(10), 677-686.

Ledesma, A. (2009). Una aproximación teórica a la relación de la empresa turística con la ética y la responsabilidad social. ROTUR/ Revista de Ocio y Turismo, (2), 125-137.

López, F., Torres, A., Font, X. \& Serrano, D. (2018). Gestión sostenible de destinos turísticos: la implementación de un sistema de indicadores de turismo en los destinos de la provincia de Barcelona. Boletín de la Asociación de Geógrafos Españoles, (77), 428-431. [En línea] Disponible en: https://bage.age-geografia.es/ojs/index.php/bage/article/view/2643 [2020, 04 de junio]

Mendizábal, G. (2013). Análisis de la responsabilidad empresarial en México de frente a los trabajadores. Revista Latinoamericana de Derecho Social, (16), 123-145.

Mendoza, M., Orgambidez, A. \& Carrasco, A. (2010). Orientación a la calidad total, satisfacción laboral, comunicación y compromiso en establecimientos de turismo rural de la Sierra de Huelva. Pasos Revista de Turismo y Patrimonio Cultural, 8(2): 351-361

Molina J., Pérez, A., Lizárraga, G. \& Larrañaga, A. (2018). Análisis de calidad de vida laboral y competitividad en empresas de servicios turísticos. 3 C Empresa: investigación y pensamiento crítico, 7(2), 44-67.

Montoya, O. (2007). Aplicación del análisis factorial a la investigación de mercados. Caso de estudio. Scientia et Technica Año XIII, (35), 281-286.

Nasrullah, N. \& Rahim, M. (2014). CSR in Private Enterprises in Developing Countries: Evidence from the ready-made garments industry in Bangladesh. In S. Idowu, \& R. Schmidpeter (eds.). Switzerland: Springer.

Organización Mundial del Turismo (OMT). (2012). Turismo y sostenibilidad. [En línea] Disponible en: http://cf.cdn.unwto.org/sites/all/files/docpdf/turismoysostenibilidad_0.pdf

Romo, A. (14 de Enero de 2016). Responsabilidad social empresarial y su evolución en México. Universidad Autónoma de Nuevo León. [En línea] Disponible en: http://eprints.uanl.mx/9733/3/RSE.pdf [2019, 20 octubre]

Salnikova, A., kovalev, A., Iosifov, V., \& Almastyan, N. (2021). Model of Circular Economy in Environmental Management. Case Study. Journal of Environmental Management and Tourism, 12(1), 5-17.

Secretaria de Turismo (SECTUR) . (28 de Octubre de 2017). Directorio de empresas con distintivo S. Secretaria de Turismo/ Documentos. [En línea] Disponible en: 
https://www.gob.mx/sectur/documentos/directorio-de-empresas-con-distintivo-s $\quad[2019, \quad 05$ diciembre]

Vélez, X. \& Cano, E. (2016). Los diferentes tipos de responsabilidad social y sus implicaciones éticas. Dominio de las ciencias: revista científica, 2, 117-126.

Viteri, J. (2010). Responsabilidad social. Facultad de Ciencias de la Ingeniería, 1, 90-100. 